\title{
Capital social y consejos de salud en Brasil. ¿Un círculo virtuoso?
}

\author{
Social capital and health councils in Brazil. \\ A virtuous circle?
}

Maria Eliana Labra 1

\footnotetext{
1 Departamento de Administração e Planejamento em Saúde, Escola Nacional de Saúde Pública, Fundação Oswaldo Cruz. Rua Leopoldo Bulhões 1480, Rio de Janeiro, $R J$ 21041-210, Brasil. labra@ensp.fiocruz.br
}

\begin{abstract}
This article begins by discussing the concept of social capital and its applications and limitations. Second, it examines these theoretical contributions as approaches actually used by scholars to analyze the disenchantment with the fragile political institutions and citizens' apathy that emerged together with re-democratization processes in Latin America. Third, it focuses on the role of the Brazilian health councils, seeking insights from this vast and rich experience of popular mobilization and participation to shed light on the councils' contributions in fostering behaviors inherent to social capital, such as civic values and cooperation.

Key words Health Councils; Consumer Participation; Health Policy
\end{abstract}

Resumen El artículo discute, en primer lugar, concepciones relativas al capital social, sus aplicaciones y limitaciones. En segundo lugar, aborda esas concepciones como recurso teórico utilizado por académicos para reflexionar sobre posibles salidas frente al desencanto y a la apatía ciudadana con las frágiles instituciones politicas que han acompañado los procesos de redemocratización en América Latina. En tercer lugar, examina los consejos de salud de Brasil buscando escudriñar en qué medida esa amplia y rica experiencia de movilización y participación popular contribuiría al fomento del capital social y, por tanto, a la adhesión a valores cívicos y a la cooperación generalizada.

Palabras clave Consejos de Salud; Participación Comunitaria; Política de Salud 
Los estudiosos del capital social han planteado que existe una relación directa entre su existencia en la sociedad y el buen desempeño de las instituciones del Estado y del mercado. Establecida esa relación, se crearía un círculo virtuoso que fomenta la confianza de los ciudadanos en las instituciones públicas e incentiva una cultura cívica de solidaridad y cooperación democrática. Como en esa visión las asociaciones comunitarias ocupan lugar central y son ellas las que se hacen representar en los consejos de salud del Brasil, es consecuente esperar que, cuanto más organizada y participativa sea la comunidad, más capital social habrá acumulado y, por lo tanto, mayor influencia tendrá en el buen cumplimiento de los objetivos del sistema de salud.

Este artículo discute esos temas en tres tópicos. Primero, examina aportes de la literatura sobre la noción de capital social, sus aplicaciones y limitaciones. Segundo, retoma esa discusión en relación con las preocupaciones contemporáneas en América Latina, con la fragilidad de las instituciones políticas surgidas de la redemocratización y con el desencanto de la ciudadanía frente a las promesas no cumplidas respecto a la resolución de sus problemas más apremiantes. Tercero, aborda los consejos de salud de Brasil, surgidos a fines de los años 80 en la senda del retorno a la democracia. Considerando que los consejos de salud constituyen una vasta y rica experiencia de movilización y participación popular que contribuye al fomento de valores y actitudes inherentes al capital social, como virtud cívica, solidaridad y cooperación, se plantea que esos cambios encuentran obstáculos no sólo en las tradiciones culturales del país sino, sobre todo, en el deficiente desempeño de las instituciones encargadas de cuidar de la salud de la población.

\section{¿Qué es capital social?}

Hay consenso generalizado en la literatura que la expresión "capital social” pasó a recibir atención especial en los medios académicos a partir de las contribuciones de Putnam (1993a, 1993b, 1996), fundamentadas en los aportes de Coleman (1990) y otros. En breve síntesis, el capital social se refiere a los sistemas horizontales de participación cívica, que pueden ser asociaciones comunitarias, orfeones, cooperativas, gremios deportivos, partidos políticos, etc. Las características centrales de esas redes de intercambio social son confianza, reciprocidad y cooperación, siendo la reciprocidad generalizada y asidua un componente altamente pro- ductivo porque facilita la solución de los dilemas de la acción colectiva (Putnam, 1996).

$\mathrm{Al}$ examinar las tradiciones de la Italia moderna del Norte y las de la Italia atrasada del Sur, Putnam deja claro que el capital social actual depende del legado histórico, de tal forma que la acumulación de experiencias exitosas de unión y cooperación entre la gente y las instituciones de gobierno reforzaron conductas de confianza recíproca, esto porque las cadenas de relaciones sociales y los recursos morales aumentan con el uso y se agotan con el desuso, concluyendo que la creación o la dilapidación del capital social caracteriza círculos virtuosos $\mathrm{y}$ viciosos.

Según Putnam (1996), el hecho de que el capital social pueda acumularse lo torna productivo, equiparándolo al capital físico y al capital humano; por tanto, cuanto más capital social acumule una sociedad, más beneficios obtendrá. Esto se debe a que los sistemas horizontales de participación cívica dan positivas contribuciones al orden social y a la mayor eficiencia de la sociedad al facilitar acciones coordenadas, debido a un mejor flujo de la comunicación e información y al posibilitar la realización de objetivos que de otra manera serían inalcanzables. Asimismo, consolidan la buena reputación, condenan el oportunismo y dificultan el clientelismo porque congregan agentes con el mismo status o poder. Como resultado virtuoso, refuerzan la confianza, la cooperación y la solidaridad entre los individuos.

En resumen, los sistemas horizontales de participación cívica llevan a crear un modelo culturalmente definido que asegura futuras colaboraciones, contribuye al mejor desempeño de las instituciones del gobierno y del mercado y fortalece la democracia. Con base a esas conclusiones, Putnam recomienda enfáticamente que los programas públicos contemplen los beneficios que podrían surgir de la creación de redes que crucen líneas sociales y raciales, pero advierte: "el capital social no es un substituto para políticas públicas efectivas, sino que es al mismo tiempo un requisito y una consecuencia de ellas" (Putnam, 1993a:42).

\section{Reconversión productiva del capital social}

Los descubrimientos de Putnam y colaboradores causaron gran revuelo entre los teóricos neoliberales de la economía por llamar la atención acerca de lo que sería "el eslabón perdido" en la cadena de causalidad entre los niveles micro y macro que conduce al desarrollo eco- 
nómico y social (Lechner, 2000). Efectivamente, el Banco Mundial realizó en 1997 un taller sobre capital social cuyos resultados fueron sintetizados por Dasgupta \& Serageldin (2000). También sobre el mismo tema, el Banco Interamericano de Desarrollo convocó en 1999 un seminario, publicándose los aportes en el compendio organizado por Kliksberg \& Tomassini (2000).

De iniciativas como esas emanaron recursos financieros para apoyar proyectos de ayuda al desarrollo en pequeñas comunidades, que incluyen la exigencia de participación y partnerships entre agencias del gobierno y la comunidad organizada, en el supuesto de que los lazos de confianza y solidaridad, así fomentados, llevarán al empowerment de los individuos y, en última instancia, al bienestar económico y social sostenido. En esa línea, Grootaert \& van Bastelaer (2002) analizaron proyectos de desarrollo en comunidades rurales a partir de tres conjuntos de medidas del capital social: la pertenencia a redes y asociaciones locales, los indicadores de confianza y adhesión a normas y la acción colectiva, llegando a la conclusión de que el capital social es un instrumento importante para reducir la pobreza y mejorar la redistribución nacional de la renta.

De hecho, desde la mitad de los años 90 se han multiplicado de forma vertiginosa los escritos e investigaciones sobre el capital social. Sin embargo, el tema es motivo de gran controversia porque, a pesar de los muchos esfuerzos, hasta ahora se carece de una definición clara. Macinko \& Starfield (2001), por ejemplo, encontraron cinco definiciones que enfatizan o el individuo, el grupo o ambos, apuntando que las evaluaciones de experiencias han mostrado resultados controvertidos. Sobre todo, se han presentado obstáculos metodológicos insalvables al medir conductas como confianza y cooperación, o las dinámicas de las redes de intercambios personales. A su vez, Durlauf (2001) desmenuzó las fórmulas econométricas empleadas en estudios empíricos del capital social con el objeto de probar las falacias de los resultados de esas investigaciones. Tanto Macinko \& Starfield como Durlauf concluyen, en resumen, que la literatura sobre el tema muestra una enorme confusión teórica, conceptual y metodológica.

En vista de resultados como esos, Norbert Lechner argumenta que el capital social funciona como una especie de "muñeca rusa" en la medida que se le van agregando atributos en un universo que permanece indefinido. Ese autor indaga sobre la enorme resonancia de la noción de capital social y concluye: “(...) la lec- tura conservadora aprecia en el concepto las virtudes de la comunidad históricamente crecida y ahora amenazada por los sistemas abstractos. El enfoque neoliberal festeja las posibilidades de una sociedad autoorganizada y autorregulada para resolver fallas del mercado sin necesidad de una intervención estatal. Los partidarios de la tercera vía visualizan la complementariedad de políticas públicas y la capacidad de asociación ciudadana. En suma, desde distintas perspectivas se ve en el capital social la oportunidad de fortalecer las capacidades de la sociedad civil" (Lechner, 2000:115).

\section{Apropiaciones del capital social en el campo de la salud}

La noción de capital social también ha invadido el área de las políticas sociales y el campo de la salud. En general, esos trabajos parten de la afirmación de Putnam de que el capital social es la goma invisible que mantiene unida a una sociedad. Desde ese punto de partida, Kawachi (1997) buscó probar que el daño derivado del debilitamiento de la cohesión social también tiene efectos físicos, llegando a la bien establecida conclusión de que un mejor nivel de salud depende de la distribución más equitativa de los ingresos.

Macinko \& Starfield (2001), por su parte, identificaron que la noción de capital social ha sido aplicada de formas variadas e incongruentes. Prueba de ello es que se la ha utilizado como: (1) un vector explicativo de la relación entre desigualdad de ingresos y nivel de salud; (2) componente de estudios sobre redes sociales y salud; (3) un mediador del desempeño de las políticas o reformas en salud; (4) sinónimo de privación o de cohesión en relación a la violencia y al crimen. Entre las investigaciones examinadas por esos autores destaca la de Rico et al. (1999), quienes relacionaron la descentralización del sistema de salud de España con indicadores de capital social y encontraron que, si bien hay relación entre buen desempeño de los gobiernos y capital social, las diferencias encontradas se debieron a la asignación inicial de recursos y al grado de autonomía de las regiones.

El capital social y los aspectos culturales que conlleva pasaron también a formar parte de las recomendaciones de las agencias internacionales de salud a los gobiernos para que incorporen tales dimensiones en la formulación e implementación de políticas. Eso ha sido notorio en el contexto del debate sobre promoción de la salud, asociándose capital social al fomento del empowerment de las comunida- 
des y a estrategias participativas. En esa línea, Restrepo (2000) considera esencial la construcción de capital social y la mejor distribución del poder para que la comunidad logre mejores niveles de salud y calidad de vida. Por otro lado, considera que la violencia en grandes áreas urbanas conduce al deterioro del capital social; para contrarrestarla, exhorta a los gobiernos a promover la cohesión social y la solidaridad, restaurando el capital social para hacer frente a la exclusión, a la desigualdad y a la opresión.

Por último, cabe mencionar el documento conceptual difundido por la Organización $\mathrm{Pa}$ namericana de la Salud (OPS, 2002) donde se discurre sobre los impactos positivos del capital social para la salud, la cultura cívica, la democracia, el entorno ambiental y el desarrollo humano, o sea, para el bienestar y la equidad de todos. De esos planteamientos derivan recomendaciones a los gobiernos para que desarrollen y fortalezcan las prácticas sociales inherentes al capital social.

En suma, la noción de capital social viene pasando por transmutaciones sucesivas y crecientes: eslabón perdido para los neoliberales, muñeca rusa para los metodólogos, velo ideológico para los infinitos males estructurales de los países de la periferia del capitalismo. A pesar de ello, en América Latina el tema ha ganado amplio espacio en los debates contemporáneos relativos a cuestiones cruciales como la precaria calidad de las nuevas democracias, la fragilidad de sus instituciones, la permanencia de rasgos patrimonialistas y clientelistas en las relaciones entre el Estado y los sectores sociales y la negligencia de los derechos de la ciudadanía. Teniendo en vista preocupaciones vitales como ésas, se ha planteado la necesidad de institucionalizar medios de participación y control popular para fortalecer la democracia. A este respecto, Brasil se destaca en el contexto latinoamericano por haber diseñado, con el advenimiento de la democracia en los años 80 y la reformulación de sus instituciones políticas, innovadoras fórmulas constitucionales para la participación indirecta y directa de los ciudadanos en la deliberación de las políticas públicas, especialmente en las áreas sociales. En ese nuevo cuadro ha sobresalido el sector salud por su liderazgo en la creación de miles de consejos de salud en todo el territorio nacional, lo cual constituye una inédita experiencia masiva de apertura de caminos para el control social "desde abajo" sobre los actos del gobierno en todos los niveles. En lo que sigue abordaremos esos temas.

\section{El capital social en la perspectiva de las nuevas democracias}

La discusión relativa al capital social ha llamado la atención hacia la importancia de la sociedad civil para el buen funcionamiento de la polis. No obstante, este tema no es nuevo en el debate filosófico, político y sociológico pues la preocupación con el buen gobierno y la buena sociedad se remonta a Platón y Aristóteles. Tal como han planteado Streeck \& Schmitter (1985), tradicionalmente se ha atribuido a la comunidad, al mercado o al Estado la capacidad de regular el conflicto. Así, la solidariedad espontánea, la concurrencia y el control jerárquico han sido paradigmas que, de forma alternada o combinada, han dominado el pensamiento social. Esos autores, además, introducen el asociativismo como una cuarta base institucional del orden social que, sumada a las demás, contribuiría a la gobernabilidad. Ese aporte vino a luz en el seno del debate sobre el neocorporativismo que Schmitter (1974) y seguidores, como Berger (1981) y muchos otros, inauguraron a propósito de las críticas a la visión pluralista del policy making predominante en las ciencias políticas en los años 1960 y 70. El tema también ganó relevancia al remitir tanto al rescate de la cuestión del Estado (Evans et al., 1985) como de la Sociedad Civil (Cohen \& Arato, 2000), sobre todo en el contexto de la pretensión neoliberal de adjudicar únicamente al mercado el rol de regulador de las relaciones sociales.

En el trascurso de esas discusiones y de la constatación de las deficiencias de las instituciones que han acompañado las redemocratizaciones en América Latina, surgieron numerosos intentos de repensar la democracia liberal no sólo como reglas del juego destinadas a asegurar la representación y participación política, tal como fue planteado por Dahl (1997, 1998), Bobbio (1987) y tantos otros. Yendo más allá, se está proponiendo "reformar" la sustancia de la democracia de modo que las instituciones puedan intervenir efectivamente en las transformaciones de la vida social y económica. Se espera, así, resolver los problemas de la gente, agravados en mucho por ajustes económicos que aumentaron el foso entre una minoría que sí goza de todos sus derechos, como en la poliarquía dahlsiana, y las mayorías crecientemente empobrecidas y excluidas de los beneficios del desarrollo. Con el agravante de que para la redistribución de la riqueza ya no se cuenta con el Estado Benefactor puesto que éste fue arrasado por la furia minimizadora que acompañó tales ajustes y por los efectos de la globalización. 
Ese repensar se acompaña de preocupaciones no menos serias ante la constatación de la existencia de nuevos valores y actitudes como escepticismo de la ciudadanía en relación a los gobiernos electos, desconfianza en la clase política, apatía participativa, fragmentación social, individualismo y particularismo, en detrimento del bienestar colectivo. Como reafirma Baquero (2001), hay consenso en cuanto a la erosión de valores ético-morales esenciales para la construcción de una real democracia social, como son la solidaridad, la amistad, la confianza recíproca en las personas y en las instituciones políticas y el declive estructural de la participación en todos los niveles de la sociedad.

Si por un lado, frente a ese cuadro de incertidumbres, se han propuesto formatos de democracias deliberativas, asociativas o participativas que complementen los procedimientos de la democracia formal, como por ejemplo, las instigadoras contribuciones de Cohen \& Rogers (1995), por otro lado, en las conceptualizaciones del capital social se han buscado elementos que inspiren la revitalización de la sociedad civil. Esto porque, como subraya Baquero (2001), en la medida que las expectativas generadas por el proceso de redemocratización han sido frustradas, el concepto de capital social puede proporcionar nuevos caminos para pensar mecanismos que den potencia a la capacidad participativa de la sociedad.

\section{La experiencia de los Consejos de Salud de Brasil}

Los Consejos de Salud (CS) representan una rica experiencia en curso muy adecuada para analizar hasta qué punto, en el ámbito sectorial, la participación de la sociedad civil organizada en esos campos está contribuyendo al fomento del capital social, o sea, a la formación de redes de intercambio basadas en lazos de solidaridad, confianza y cooperación que, en última instancia, coadyuvan a la adquisición de valores cívicos y al fortalecimiento de la democracia. Dicha experiencia surge y se desenvuelve en un medio muy adverso, dadas las profundas desigualdades económicas, sociales, raciales, de género y territoriales que marcan la sociedad brasileña, agudizadas por las intermitentes crisis del capitalismo global que con exorbitante fuerza afectan al país, privándolo de cruciales recursos para paliar las deficiencias en las áreas sociales.

En breve síntesis, los primeros movimientos sociales urbanos surgieron en los años 40 , cuando pobladores se organizaron en torno del problema de la vivienda. Ese asociativismo, posteriormente diseminado, se sumó en los años 80 a las denodadas luchas por la democratización del país y de la salud, convergiendo hacia un importante movimiento nacional que empuñó banderas reivindicativas de cambios radicales en el caótico, ineficiente e insuficiente sistema de salud existente. De esa forma, los CS fueron producto mediato de la revitalización de la sociedad civil durante el proceso de democratización del país y una derivación directa de las exigencias en pro de la salud como un derecho universal a ser garantizado por el Estado. Como resultado de esas presiones, con la Constitución Federal de 1988 se creó el Sistema Único de Salud (SUS), cuyas leyes orgánicas establecieron la creación de un Consejo Nacional de Salud y consejos en cada estado y municipio del país, acompañando, así, el proceso descentralizador involucrado en la implementación de la nueva institucionalidad.

Es interesante notar que la composición de los CS debe guardar la paridad entre los representantes de los usuarios de los servicios y de las autoridades, proveedores públicos y privados y trabajadores del sector. El objetivo primordial de la presencia de los usuarios es que participen de las deliberaciones sobre las políticas y los programas de salud y ejerzan el control social sobre los actos de las autoridades locales destinados a implementar las directrices decididas. Cabe resaltar que formar parte de la dinámica de los CS la realización de conferencias de salud a cada cuatro años en todo el territorio nacional y que culminan en la Capital Federal, donde cerca de 4 mil consejeros discuten de forma abierta y democrática asuntos relativos al SUS, a la atención de la salud y al control social.

Los CS comenzaron a ser implantados en el año 1990. Actualmente existen más de 5.500 colegiados y cuentan con respaldo de todos los sectores involucrados. El examen de esas áreas ha mostrado que entre sus miembros existe alto grado de cooperación y que las discrepancias son resueltas mediante negociaciones y consensos (Silva \& Labra, 2001). Sin embargo, el funcionamiento de los CS no es homogéneo en el país, presentando en muchas localidades dificultades que pueden ser atribuidas a varios factores, de los cuales a continuación se señalan los principales con base en trabajos de investigación bibliográfica y de campo de Figueiredo (2001), Labra (2002) y de Labra \& Figueiredo (2002).

En primer lugar, aún no se ha resuelto del todo la cuestión de la representatividad de los 
consejeros pertenecientes al segmento de los usuarios debido a interferencias políticas o clientelistas en su nombramiento, de modo que a menudo esos personeros no representan los intereses de los ciudadanos comunes. Por otra parte, el reglamento de los CS dispone que el secretario local de salud, o gestor, los presida. Entre tanto, muchos gestores actúan de forma autoritaria al no acatar las resoluciones de los consejeros, recurriendo a expedientes como manipulación, coacción u omisión, para conseguir imponer sus propias determinaciones. A ello contribuye en el contenido de los temas abordados en los CS, puesto que, en general, son técnicamente complejos y requieren conocimientos especializados que los legos no dominan. Dígase de paso que este problema está siendo paliado con cursos de capacitación como el que se realiza actualmente para $43 \mathrm{mil}$ consejeros. No obstante, cabe mencionar que debido al hecho de que los miembros de los CS deben renovarse cada dos años; y dado que los nuevos consejeros no tienen ese entrenamiento, el esfuerzo en este sentido puede ser en vano. Otro punto que dificulta el avance de los trabajos de los CS es la frecuencia mensual de las reuniones. Esta periodicidad se ha mostrado insuficiente para encargarse de una agenda sobrecargada por los asuntos previstos y los urgentes que siempre emergen. A esto se le une el hecho de que muchos CS de las regiones metropolitanas cubren extensas áreas geográficas con una población que puede exceder un millón de habitantes, lo cual imposibilita un conocimiento cabal tanto de los problemas y necesidades de salud de la población del área como de los programas y servicios para atenderlos. Por último, hay que referirse al control social, que es el objetivo primordial de los CS pero se ha convertido prácticamente en una metáfora. En efecto, un expediente fundamental que debe utilizar el gestor para materializar el control social es someter al colegiado el presupuesto local anual y, al año siguiente, dar cuenta comprobada de su realización, conforme el plan de acción también aprobado previamente. Sin embargo, salvo raras excepciones, los gestores no cumplen esas exigencias, lo cual genera en los consejeros sentimientos de frustración.

Con respecto a la representación de los usuarios, en la Región Metropolitana de Río de Janeiro se constata que más del $70 \%$ de los integrantes de ese segmento actúa en representación de las asociaciones de vecinos de barrios y de favelas, las cuales los eligen o indican con base en criterios como facilidad de palabra, carisma y experiencia en trabajo comunitario o en otros consejos. El perfil de esos con- sejeros, a su vez, presenta un nivel educacional y socioeconómico superior a la media de los habitantes de la Región y predominan representantes del sexo masculino. Otro aspecto que llama la atención es la baja rotación de esos consejeros, posibilitada por sucesivas reelecciones, aunque esto puede tener relación con el tiempo que la persona necesita para familiarizarse con los temas de los CS, lo que apuntaría hacia una profesionalización de la función de consejero y, sin embargo, podría ser también un indicador de la reproducción de las relaciones jerárquicas, clientelistas y excluyentes que se observan en la sociedad como un todo, puesto que, como veremos adelante, las elecciones de dirigentes y representantes es un proceso poco claro.

Por otro lado, fue constatado que la mayoría de esos consejeros tiene alto aprecio por su función, señalando inclusive que la participación en el CS les llevó a preocuparse por cuestiones relacionadas con el gobierno local y el interés general, además de haberlos incentivado a mantenerse informados y a elevar su nivel educacional y cultural, o sea, les llevó a adquirir valores cívicos y meritorios.

En relación a la dimensión asociativa, no cabe duda que los CS han sido un motor fundamental para la activación y crecimiento de las organizaciones de vecinos y la rápida creación de agrupaciones de portadores de patologías y deficiencias, que son muy activas. No obstante, analizada la constelación de asociaciones de vecinos a la luz de los componentes del capital social, los resultados no son muy alentadores. En varios puntos de las investigaciones realizadas fue constatado que aquellas agrupaciones tienden a ser manejadas por un pequeño grupo que se perpetúa en el comando y recurre a artimañas para no abrirse a la comunidad, como señalaría la exigencia de estar en día con la cuota mensual de afiliación (cerca de 70 centavos de dólar), para participar en las reuniones de la directiva y en las elecciones de ésta y de consejeros, entre otras actividades que requerirían la presencia de la comunidad. Para los dirigentes, la explicación está en la apatía de la gente, que no participa porque sólo se preocupa de sus problemas personales, inmediatos y concretos.

En todo caso, la forma en que funcionan las asociaciones de vecinos ratifica que en sus relaciones con las comunidades permanecen rasgos autoritarios y elitistas observados en la cultura nacional, inclusive en las regiones más carentes que son, justamente, las que más deberían mostrar mayores grados de unión y participación. 
Con respecto a la situación de la atención a la salud, tanto de nuestras indagaciones como de las de otros estudiosos del tema, como Lopes \& Almeida (2001) y Lima (2001), surge la opinión generalizada de que aún no se han resuelto problemas básicos, como las demoradas listas de espera por una consulta, el via crucis para conseguir una internamiento hospitalario, la falta de médicos y de medicamentos, el poco respeto de funcionarios con pacientes humildes y, de particular importancia en Río de Janeiro por el cuadro de violencia que vive, la carencia de servicios de urgencia.

Se debe acotar que la implementación del SUS es una iniciativa de una extraordinaria complejidad y de altísimo costo. Se han alcanzado grandes avances en materia de municipalización y consiguiente traspaso de responsabilidades y recursos a los niveles locales. No obstante, esos esfuerzos al parecer aún no son perceptibles a ojos de la población. Esto también se refleja en los CS, que han servido principalmente para encaminar demandas por más y mejor asistencia médica. En verdad, en la medida que la resolución de los enormes y múltiples problemas del SUS no es tangible o visible para el público, las peticiones por más de lo mismo se reiteran ad infinitum. Y esto tiene consecuencias para los CS ya que, frente a la expectativa del aplazamiento de sus demandas para un futuro que nunca llega, se corre el riesgo de esterilizar esas áreas debido a la frustración e impotencia que acarrea el bajo retorno que la movilización y la participación tiene. Un ejemplo que ilustra esta afirmación es la baja calidad de la atención al embarazo y al parto: en el estado de Río de Janeiro la mortalidad materna se ha mantenido muy alta durante toda la década de 90 , en torno de 80 óbitos por cien mil nacidos vivos (SES-RJ, 1999), cuando el ideal sería menos de 10 óbitos. Esas tasas pueden ser atribuidas a la mala distribución territorial de la red asistencial que en sí es densa y bien dotada de recursos y tecnologías pero también se debe considerar la calidad de la atención, que deja mucho a desear, o por lo menos no ha mejorado de forma ostensiva con el SUS.

Dados esos antecedentes, puede decirse que la relación sinérgica que, según Putnam (1996), existiría entre la existencia de capital social y desempeño institucional aún está lejos de evidenciarse en la salud; al contrario, se podría esperar que la laboriosa y dedicada participación de ciudadanos en los CS termine por convertirse en simple formalidad ya que los consejeros no tendrían incentivos para mantener la cooperación. Los CS no pueden dejar de existir porque por ley son requisito para que los gestores estén habilitados a recibir los recursos financieros que el Ministerio de Salud les transfiere en función de los diversos programas nacionales que ha fijado. En vista de eso, los consejeros representantes de los usuarios podrán encontrar otros acicates para pactar con el ritualismo de una participación que sí podrá dar otros dividendos como mayor prestigio social, influencia y poder frente a la comunidad, el logro de un status social que facilita el acceso a los políticos al momento de buscar otros beneficios e, incluso, hacer uso del cargo para conseguir una atención más rápida en los establecimientos de salud.

Por último, se debe hacer mención a las alternativas que la población carente viene encontrando para enfrentar las múltiples omisiones y deficiencias del Estado en la provisión normal de bienes y servicios públicos. Se trata de nuevas experiencias de alianzas formales que, al estilo de lo recomendado por las agencias internacionales, las asociaciones de vecinos han estado estableciendo con la alcaldía de Río de Janeiro para atender a necesidades básicas, como son las de salud. En este caso, estudiado por Claro (2002), el ejemplo se refiere a la Organización No Gubernamental que crearon 17 asociaciones comunitarias de una favela con cerca de 300 mil habitantes y enormes problemas, para instalar un puesto básico de salud, entre otras iniciativas. La sociedad con la secretaría municipal de salud contempla dos componentes principales: el traspaso de recursos financieros mediante convenio y la gestión del puesto por parte de la comunidad. Se trata de un trabajo muy mal remunerado, que también cuenta con voluntarios en la organización. La atención es, por definición, muy elemental pero sirve para cuidados de rutina, como retaguardia de la vigilancia que agentes comunitarios de salud efectúan en los domicilios y para encaminar pacientes a los hospitales en caso necesario. De todas formas, esas iniciativas, que requieren esfuerzos cotidianos inauditos de los pobladores para llevarlas adelante, son proyectos pobres para pobres: son en verdad mínimos, dependen de recursos financieros fiscales siempre insuficientes, que demoran en llegar, y cuya continuidad es incierta porque está sujeta a la voluntad política de los gobernantes de turno.

Los ejemplos señalados reiteran que el fomento al capital social también depende de las condiciones que las autoridades ofrezcan para ello. En realidad, la alianza entre la permanencia de elementos culturales tradicionales y el deficiente desempeño institucional, no sólo en la salud, sino también en los demás campos sociales y en áreas como la justicia y la seguri- 
dad ciudadana, estaría contribuyendo al incremento de la desconfianza de la ciudadanía en las instituciones públicas, en los políticos y en la democracia.

\section{A modo de conclusión}

La apreciación crítica del concepto denominado "capital social" ha mostrado su gran elasticidad y ambigüedad. A pesar de ello, señala Baquero (2001), para los estudiosos de los problemas de las democracias latinoamericanas contemporáneas, ha venido a servir como un puente entre el mundo teórico y el mundo real. Uno de los elementos de esa categoría, que ha generado entusiasmo tiene que ver con su dimensión lógica en la explicación de problemas sociales de la actualidad. Por ejemplo, se ha diseminado la idea de que una sociedad que exhibe padrones tradicionales de comportamiento político (clientelismo, personalismo e patrimonialismo) y que convive con la corrupción institucionalizada, puede ser explicada en gran parte por el hecho de que los ciudadanos no han invertido suficientemente en la acumulación de capital social (Baquero, 2001).

Esta exposición ha apuntado en una dirección que difiere de esos planteamientos. A pesar de la semejanza de las tradiciones culturales de Brasil con las señaladas arriba, de sus enormes desigualdades y de la pobreza de la mayoría de sus habitantes, muchas experiencias se vienen acumulando en términos de participación social organizada en los múltiples espacios públicos creados para ello. El más famoso de todos, y que omitimos por razones de brevedad, es el Presupuesto Participativo de Porto Alegre, que ha ganado repercusión internacional. Sin embargo, los CS son igualmente dignos de destacar, ya que involucran a miles de personas, están presentes en los lugares más recónditos del país y se traducen en una acción colectiva de gran envergadura. A pesar de que el funcionamiento de los CS presenta diversas dificultades, poco a poco están siendo subsanadas en la medida que se ha formado una masa crítica dispuesta a luchar por su perfeccionamiento. Entre tanto, vencer el autoritarismo de los gestores y su reluctancia en someter las cuentas públicas al colegiado, para que así ejerza el control social, va a ser, ciertamente, el desafío mayor de todos.

La participación en los CS ha requerido ingentes esfuerzos de movilización y de organización de la población, así como la dedicación altruista de los representantes de las asociaciones de vecinos. Por otra parte, es cierto que las organizaciones populares presentan rasgos oligárquicos y procesos dudosos para elegir dirigentes y representantes en los CS. Además, la distancia entre esos representantes y los usuarios de los servicios de salud es enorme debido, en buena medida, a que la instalación de los colegiados acompaña la división territorial del sector salud en las grandes ciudades y esas áreas tienen, en general, elevado número de habitantes. Todas esas dificultades y otras ya mencionadas en relación con el funcionamiento de los CS y de las organizaciones comunitarias son serias pero en gran medida superables. No sucede así con las tendencias señaladas en conexión con el desempeño de las instituciones de la salud.

A este respecto, cabe recurrir a Putnam (1993a:42) cuando advierte que "el capital no es un substituto para políticas públicas efectivas sino que es al mismo tiempo un requisito y una consecuencia de ellas". De hecho, en el sector salud se han creado todas las condiciones para el establecimiento de redes de confianza, solidaridad y cooperación entre los ciudadanos y éstos se han mostrado dispuestos a participar con entusiasmo de los CS y de los demás eventos que se promueven para mantener viva la llama de la democratización del SUS. No obstante, esta acumulación de capital social no es por si sóla suficiente para provocar el círculo virtuoso de solidaridad, confianza y cooperación, señalado por Putnam, como producto de la unión entre la gente y las instituciones de gobierno. En realidad, la experiencia brasileña en la salud muestran que es conditio sine qua non la reciprocidad de los poderes públicos en esas relaciones de intercambio con los ciudadanos.

En efecto, en la medida que las políticas de salud son poco efectivas, los servicios funcionan mal, los gestores son autoritarios o displicentes y el control social es coartado, los efectos pueden ser opuestos a lo esperado por los teóricos del capital social: perpetuación de valores culturales tradicionales, ritualización de la representación de los usuarios en los CS, falta de compromiso de los consejeros frente a la comunidad y comportamientos individualistas. Todo eso redundaría, inversamente, en un círculo vicioso que fomenta la apatía participativa, la desconfianza en los poderes públicos y en los políticos, el descrédito, en fin, de la democracia y sus instituciones. 


\section{Referencias}

BAQUERO, M., 2001. Alcances e limites do capital social na construção democrática. In: Reinventando a Sociedade na América Latina (M. Baquero, org.), pp. 19-49, Porto Alegre: Editora da UFRGS/ Brasília: Conselho Nacional dos Direitos da Mulher.

BERGER, S. (ed.), 1981. Organized Interests in Western Europe. Cambridge: Cambridge University Press.

BOBBIO, N., 1987. O Futuro da Democracia. Uma Defesa das Regras do Jogo. 3a Ed. Rio de Janeiro: Paz e Terra.

CLARO, I. B., 2002. Gestão Comunitária: Estudo de uma Nova Articulação Estado/Sociedade Civil no Parque Proletário de Vigário Geral. Dissertação de Mestrado, Rio de Janeiro: Escola Nacional de Saúde Pública, Fundação Oswaldo Cruz.

COHEN, J. L. \& ARATO, A., 2002. Sociedad Civil y Teoría Política. México, DF: Fondo de Cultura Económica.

COHEN, J. L. \& ROGERS, A., 1995. A proposal for reconstructing democratic institutions. In: Associations and Democracy (E. O. Wright, ed.), pp. 7-98, London/New York: Verso.

COLEMAN, J., 1990. Foundations of Social Theory. Cambridge: Harvard University Press.

DAHL, R. A., 1997. Poliarquia. Participação e Oposição. São Paulo: Edusp.

DAHL, R. A., 1998. On Democracy. New Haven/London: Yale University Press.

DASGUPTA, P. \& SERAGELDIN, I., 2000. Social Capital. A Multifaceted Perspective. Washington, DC: World Bank.

DURLAUF, S. N., 2001. On the Empirics of Social Capital. Cambridge: Department of Economics, University of Wisconsin/National Bureau of Economic Research/Summer Institute. (mimeo.)

EVANS, P.; RUESCHMEYER, D. \& SKOCPOL, T. (ed.), 1985. Bringing the State Back In. Cambridge: Cambridge University Press.

FIGUEIREDO, J., 2001. Comunidade Cívica, Capital Social e Conselhos de Saúde no Estado do Rio de Janeiro. Dissertação de Mestrado, Rio de Janeiro: Escola Nacional de Saúde Pública, Fundação Oswaldo Cruz. Rio de Janeiro.

GROOTAERT, C. \& van BASTELAER, T., 2002. Understanding and Measuring Social Capital. Washington, DC: United States Agency for International Development/Center for Institutional Reform and the Informal Sector. (mimeo.)

KAWACHI, I., 1997. Long live community. Social capital as public health. American Prospect, 8(35). < http: //www.prospect.org/print/V8/35/kawachi-i. html>.

KLIKSBERG, B. \& TOMASSINI, L., 2000. Capital Social y Cultura: Claves Estratégicas para el Desarrollo. Buenos Aires: Banco Interamericano de Desarrollo/Fondo de Cultura Económica de Argentina.

LABRA, M. E., 2002. A Qualidade da Representação dos Usuários nos Conselhos de Saúde e a Dimensão Associativa. Relatório Final de Pesquisa. Rio de Janeiro: Escola Nacional de Saúde Pública, Fundação Oswaldo Cruz.

LABRA, M. E. \& FIGUEIREDO, J., 2002. Associativismo, participação e cultura cívica. O potencial dos
Conselhos de Saúde. Ciência \& Saúde Coletiva, 7:537-547.

LECHNER, N., 2000. Desafíos de un desarrollo humano: Individualización y capital social. In: Capital Social y Cultura: Claves Estratégicas para el Desarrollo (B. Kliksberg \& L. Tomassini, org.), pp. 101-127, Buenos Aires: Banco Interamericano de Desarrollo/Fondo de Cultura Económica de Argentina.

LIMA, J. C., 2001. Representatividade e participação das bases na categoria dos usuários de um Conselho Municipal de Saúde. Saúde em Debate, 25: 16-28.

LOPES, M. L. \& ALMEIDA, M. J., 2001. Conselhos Locais de Saúde em Londrina (PR): Realidade e desafios. Saúde em Debate, 25:29-39.

MACINKO, J. \& STARFIELD, B., 2001. The utility of social capital in research on health determinants. Milbank Quarterly, 3:387-427.

OPS (Organización Panamericana de la Salud), 2002. La Salud Pública en las Américas. Documento Conceptual. Washington, DC: OPS. (mimeo.)

PUTNAM, R., 1993a. The prosperous community: Social capital and public life. American Prospect, 13:35-42.

PUTNAM, R., 1993b. Making Democracy Work: Civic Traditions in Modern Italy. Princeton: Princeton University Press.

PUTNAM, R., 1996. Comunidade e Democracia. A Experiência da Itália Moderna. Rio de Janeiro: Editora FGV.

RESTREPO, H. E., 2000. Increasing Community Capacity and Empowering Communities for Promoting Health. Fifth Global Conference on Health Promotion. Mexico, DF: Pan American Health Organization. (mimeo.)

RICO, A.; FRAILE, M. \& GONZALEZ, P., 1999. Regional decentralization of health policy in Spain: Social capital does not tell the whole story. West European Politics, 21:180-99.

SCHMITTER, P. C., 1974. Still the century of corporatism? Review of Politics, 36:85-131.

SES-RJ (Secretaria de Saúde do Estado do Rio de Janeiro), 1999. Relatório do Comitê de Mortalidade Materna e Infantil. Rio de Janeiro: SES-RJ.

SILVA, I. \& LABRA, M. E., 2001. As instâncias colegiadas do SUS no Estado do Rio de Janeiro e o processo decisório. Cadernos de Saúde Pública, 17: 161-170.

STREECK, W. \& SCHMITTER, P. C., 1985. Community, market, state - and associations? The prospective contribution of interest governance to social order. In: Private Interest Government. Beyond Market and State (W. Streeck \& P. C. Schmitter, ed.), pp. 1-29, London: Sage.

Recibido el 4 de junio de 2002

Versión final presentada el 19 de septiembre de 2002 Aprobado el 15 de octubre de 2002 